\title{
Systemic Lupus Erythematosus Presenting as Cardiac Tamponade Successfully Treated With High-Dose Steroid: A Case Report
}

\author{
Mariama Akodad, Marion Berous, Benoît Lattuca, Sébastien Jugant, François Roubille
}

Mariama Akodad, Marion Berous, Benoît Lattuca, Sébastien Jugant, François Roubille, Intensive care unit department, Montpellier University Hospital, France

Correspondence to: François Roubille, MD, PhD, Intensive care unit department, Montpellier University Hospital, France

Email: francois.roubille@gmail.com

Telephone: $+33-473751410 \quad$ Fax: $+33-473754814$

Received: February 11, 2015 Revised: March 6, 2015

Accepted: March 12, 2015

Published online: June 10, 2015

\begin{abstract}
We report a case of a young woman with systemic lupus induced cardiac tamponade. After discussion with the cardiac surgeons' team, medical management was decided and no pericardiocentesis was performed. Bolus of Methylprednisolon intravenously were administrated with a rapid improvement of the symptom and resolution of the pericardial effusion.
\end{abstract}

C 2015 ACT. All rights reserved.

Key words: Cardiac tamponade; Pericardial effusion; Systemic lupus erythematosus; Steroid

Akodad M, Berous M, Lattuca B, Jugant S, Roubille F. Systemic Lupus Erythematosus Presenting as Cardiac Tamponade Successfully Treated With High-Dose Steroid: A Case Report. Journal of Cardiology and Therapy 2015; 2(3): 336-337 Available from: URL: http://www.ghrnet.org/index.php/jct/article/view/1213

\section{CASE REPORT}

A 29-year-old woman presented to the emergency department for chest pain and dyspnea.

She had a 4-year history of Lupus Erythematosus associated with AntiPhospholipid Antibody syndrome revealed by a pulmonary embolism.

She presented recurrent polyarteritis episodes since the diagnosis of lupus erythematosus, with the last one 6 months before the index hospitalization. All the recurrences had been treated successfully with high doses of Methylprednison and the disease was considered controlled.

Her usual medication was Prednisone $10 \mathrm{mg}$ one daily and Fluindione $15 \mathrm{mg}$ daily with a correct observance.

At the admission, the hemodynamic was stable with a blood pressure of $106 / 57 \mathrm{~mm} \mathrm{Hg}$, heart rate of 110 beats/min, oxygen saturation of $98 \%$ on room air and temperature of $37.2^{\circ}$. Physical examination showed soft leg edemas and decreased breath sounds on the left.

The 12- lead electrocardiogram was considered as normal with a physiologic incomplete right bundle branch block and a 120 beats/ min sinusal tachycardia.

An echocardiogram was logically performed and revealed a large pericardial effusion with multiple partitions and thick aspect.

The maximal diameters of this effusion were of $27 \mathrm{~mm}$ compared to the left ventricle and $10 \mathrm{~mm}$ to the right, with diastolic collapse of right atrium and right ventricle suggesting a cardiac tamponade. A moderate left pleuritis was also observed on pleural echography.

The blood cell count showed elevated neutrophilia and C-reactive protein was raised at $245 \mathrm{mg} / \mathrm{L}$. The International Normalized Ratio (INR) was in therapeutic window.

Pericardiocentesis was estimated to be technically difficult given a largely posterior fluid collection and the hemorrhagic risk induced by anticoagulant therapy.

Surgical approach was considered but seemed too invasive regarding to the relative clinical good tolerability.

The rheumatology service was then consulted and their recommended to initiate therapy with Methylprednisolone $100 \mathrm{mg}$ intravenously once per day during 3 days under a close monitoring of the patient assessing for response to the treatment. The decision was guided by the previous successful responses to the high dose steroid during the recurrent episodes of arthritis.

The oral anticoagulation was stopped and replaced by 
intravenous heparin because of a plan to perform emergent pericardiocentesis if any deterioration occurred.

Significant clinical improvement occurred 3 days later and the intravenous treatment was followed by Prednisone $30 \mathrm{mg}$ by mouth daily.

Repeated transthoracic echocardiography 5 days after the initial study demonstrated no residual pericardial or pleural effusion. The C-reactive protein level gradually decreased until $34 \mathrm{mg} / \mathrm{L}$.

The patient was discharged under the same dose of prednisone with instruction of close follow-up and to perform an echocardiography at 1 month.

Pericarditis is a frequent cardiac manifestation of systemic lupus erythematosus (50-62\%) but tamponade -although classical- is scarcely reported $(1 \%)^{[1,2]}$

We present a rare case of lupus erythematosus presenting with isolate cardiac tamponade resolved with administration of highdose steroids although pericardiocentesis is frequently associated in the literature ${ }^{[2]}$.

Initiation of steroid therapy should be performed promptly and may be sufficient. Under close monitoring, medical treatment should be considered in selected patients with probability of favorable response to steroid and hemodynamic stability presenting lupus erythematosus related pericardial effusion.

\section{CONFLICT OF INTERESTS}

There are no conflicts of interest with regard to the present study.

\section{REFERENCES}

1. Kahl LE. The spectrum of pericardial tamponade in systemic lupus erythematosus. Report of ten patients.Arthritis Rheum. 1992;35(11):1343-1349. doi: 10.1002/art.1780351115

2. Rosenbaum E, Krebs E, Cohen M, Tiliakos A, Derk CT. The spectrum of clinical manifestations, outcome and treatment of pericardial tamponade in patients with systemic lupus erythematosus: a retrospective study and literature review. Lupus. 2009;18:608-12. doi: $10.1177 / 0961203308100659$

Peer reviewer: Mikhail Betekhtin, Moscow multiprofile medical center, Department of dermatovenereology, Moscow, 111123, Russia. 